\title{
TCP Throughput Efficiency Enhancement In IEEE 802.11n Network
}

\author{
Onabajo Olawale Olusegun \\ Faculty of Computer Science \& IT \\ Universiti Malaysia Sarawak \\ 94300 Kota Samarahan, Malaysia \\ victorious_gem@yahoo.com
}

\author{
Chong Eng Tan \\ Faculty of Computer Science \& IT \\ Universiti Malaysia Sarawak \\ 94300 Kota Samarahan, Malaysia \\ cetan@ieee.org
}

\begin{abstract}
The new opportunities opened up by wireless technologies are also accompanied with new technical challenges. Principal among the challenges is the fact that wireless medium has limited bandwidth resources when compared with wired equivalent. The convenience brought about by the IEEE 802.11 n protocol is also followed by the technical challenge of near average performance of the widely used transport control protocol (TCP) due to limited bandwidth resources of the wireless medium. IEEE 802.11n specifies standard for physical (PHY) and MAC layers of wireless local area networks (WLANs) which is based on the IEEE 802.11-2007 networking reference standard. This work is meant to enhance the efficiency of TCP in IEEE 802.11n network in order to improve per user bandwidth. ACK suppression in both downstream and upstream TCP flow is used to improve bandwidth. The network bandwidth of an IEEE 802.11n LAN is simulated under two access methods; request-tosend/clear-to-send (RTS/CTS) access method and the basic method. Results showed an improvement in throughput efficiency over 10 nodes, which demonstrate performance improvement of TCP ACK suppression as $50 \%$ in comparison with receiver policy of acknowledging every TCP data segment and approximately $20 \%$ when compared with receiver's policy of acknowledging every TCP data segment in the network. Simulation was conducted in Matlab R2012a.
\end{abstract}

\section{Keywords-IEEE 802.11n; Throughput; SNR; TCP}

\section{INTRODUCTION}

The advancement in multimedia applications and the growth of the Internet has brought about the demand for highspeed digital communication systems. Sophisticated audio and video coding methods have reduced bit rate requirements for audio and video transmission. This in turn motivated the development of communication systems to achieve these requirements; brought technologies enabled high-quality audio and video transmission and introduced a number of new applications for businesses and residential consumers.

Current work investigates the problem of drastic fall in bandwidth with increasing distance. This paper focuses on improvement of bandwidth delivered over long distances using TCP throughput efficiency enhancement of IEEE $802.11 \mathrm{n}$ network. Most rural villages in developing world exist in clusters, with considerable distance between them ranging from $4-10 \mathrm{~km}$ or even more [1]. Long distance from network backbone results in bandwidth attenuation, therefore extremely remote rural villages end up with relatively low bandwidth incapable of supporting basic network services such as email, and voice telephony.

In recent past, the most powerful set of specifications currently deployed is the IEEE $802.11 \mathrm{~g}$ radio which operates on a bandwidth of $20 \mathrm{MHz}$ and its Physical Layer (PHY) is based on a particular form of multicarrier transmission, namely, orthogonal frequency division multiplexing (OFDM) which enables these systems to achieve transmission rates of up to $54 \mathrm{Mbps}$. However, bandwidth intensive applications has made IEEE $802.11 \mathrm{~g}$ data rate insufficient, and thus made way for a superior one. At present, a new standard IEEE $802.11 \mathrm{n}$, is currently gaining ground because of its higher data rate. PHY channel extension to IEEE 802.11-2007 from $20 \mathrm{MHz}$ to 40 $\mathrm{MHz}$, the addition of Multiple Input Multiple Output (MIMO) features, and frame aggregation at the Media Access Control (MAC) layer gave birth to IEEE $802.11 \mathrm{n}$ in 2009 [2].

To alleviate the problem of drastic fall in bandwidth delivered over long distance, this work proposed TCP efficiency enhancement of IEEE 802.11n network. In meeting this goal, a simulation model of IEEE 802.11 n was setup in Matlab to simulate different scenarios of network topology that represents the environment under investigation. Results obtained confirmed improvement in network bandwidth.

\section{BACKGROUND}

WLANs have evolved from an interesting idea to an essential and important technology that many individuals and businesses cannot live without today. The first Wi-Fi standards in the 802.11 series were produced in 1999 [3]. The next two standards produced by the IEEE were $802.11 \mathrm{~g}$, in 2003 and $802.11 \mathrm{n}$ in 2009. Each attempt is to improve on the performance of $802.11 \mathrm{~b}$ and give it the performance achieved by $802.11 \mathrm{a}$ without raising the cost out of range of the consumer market. As users of network resources, we always ask for more; we need more speed and coverage. Under this circumstance, it then built up the foundation for the next 